pas à considérer ici si c'est à tort ou à raison; ce qui importe uniquement, c'est que la description du subulatus Bris. s'applique exactement au Ceuthorrhynchus trouvé à Montpellier.

Ceuthorrhynchus subulatus Bris. se trouve à Montpellier, en mai, sur une Labiée à fleurs roses, Phlomis Herba-venti L. ${ }^{1}$ ).

Ce charancon n'est pas rare, mais parait très localisé. En effet, Phlomis Herba-venti L. est une plante assez répandue sur le bord des chemins de toute la plaine et des basses montagnes du département de l'Hérault, et cependant je n'ai jamais trouvé Ceuthorrhynchus subulatus Bris. qu'à un seul endroit, à trois kilomètres à l'Est de la ville, près du ruisseau de la Lironde, au bord d'un sentier où poussent cing ou six pieds de Phlomis Herba-venti. Mes recherches en d'autres lieux sont restées infructueuses.

L'insecte ronge les bourgeons terminaux des tiges avant la floraison. Les bourgeons sont souvent complètement dévorés par les Ceuthorrhynchus, qui se tiennent soit sur le bourgeon, soit cachés entre ses jeunes feuilles non encore épanouies. J'en ai observé parfois plus d'une dizaine sur un même bourgeon.

Je ne veux pas terminer cette note sans remercier MM. H. DU Bursson et A. Hustache de l'aide qu'ils ont bien voulu me donner pour la détermination de cette espèce.

\title{
Chrysopides nouveaux [NEVR.]
}

par J. LACROIX.

Ghrysopa gallica, n. sp. - Similis C. flavifronti Brauer. Caput flavum, stria semilunari ante antennas et puncto laterali ad occiput juxta oculos, rubris; stria nigra ad clypei latera; palpis nigris, ad articulationes flavis; oculis in sicco plumbeis. Antennis flavis, apicem versus fuscescentibus. Prothorax transversus, viridi-flavus, superne fascia longitudinali ad margines laterales fusco-rubra. Mesothorax et metathorax virides. Abdomen viride, flavido pilosum, inferne pallidius. Pedes viridi-pallidi, fusco pilosi, genibus tarsisque rufescentibus. Alae grandes, apice acutae, hyalinae; reticulatione et stigmate viridi-albis ; pilis fuscis, longiusculis; fimbriis flavis. Ala anterior venulis grada-

(1) Celte espèce a été déjà observée en Algérie sur le même Phlomis par M. P. De Peyerimhoff. 
tis $6 / 8$ totis, costalibus initio et fine, plerisque reliquis initio tantum nigris. Venulae intermediae quatuor, prima ad quartum apicale cellulae procubitalis typicae inserta.

Ala posterior venulis gradatis fere $5 / 8$ tenuibus, costalibus et aliquot juxta basim totis, radialibus initio nigris.

Longueur du corps 8 mill., de l'aile antérieure $10 ̈$ mill., postérieure 13 mill.

Habitat, : Saint-Martin-de-la-Coudre (Charente-Inférieure). 18 juin 1912. Le type dans ma collection.

Ghrysopa Navasi, n. sp. - Viridis. Caput viridi-flavum; stria semilunari ante antennas, medio se contingente, alia brevi transversa pone antennas, duobus punctis in vertice et alio ad occiput juxta oculos, rubris. Stria grandi nigra ad genas. Palpis subtotis nigris. Antennis flavis. Oculis in sicco fuscis. Prothorax latior quam longior, viridis, fascia fusca laterali ad margines. Mesothorax et metathorax viridia; puncto laterali ad proscutum et ad scuta fusco-rubro. Abdomen viride viridi pilosum, immaculatum. Alae angustae, irideae; reticulatione viridi; stigmate viridi; pilis fuscis; puncto fusco pone costam ad basim. Ala anterior apice elliptice rotundata; venulis costalibus, ultimis radiulibus, primis intermediis, gradatis $4 / 6 \mathrm{vel} \mathbf{5} / 6$, procubitalibus, cubitalibus et marginalibus posterioribus totis, reliquis et axillis furcularum marginalium initio et fine, sectore radii ejusque ramis initio tantum, nigris. Venula intermedia prima ad tertium apicale cellulae procubitalis typicae deveniens.

Ala poterior apice acuta, pallidior; venulis costalibus, gradatis $4 / \overrightarrow{5}$ et aliquot juxta alae basim totis, reliquis plerisque ad utrumque apicem nigris.

Longueur du corps 6 mill. 5̆, de l'aile antérieure 11 mill. 4, postérieure 10 mill. 4.

Habitat : Falicon (Tunisie) 16 juillet 1912, Daniel Lucas. Le type dans ma collection.

Je me fais un devoir et un plaisir, en témoignage de ma profonde reconnaissance, de dédier cette espèce à mon éminent maitre le $\mathrm{B}$. P. Longinos Navas.

Chrysopa Mariana Navas, var. insignata, n. var. - Abdominis secundum, tertium, quartum, quintum sextumque segmentum ferunt, in dorso, quatuor puncta pulla; quorum duo sunt in anteriore parte, duo in posteriore.

In ala superiore venulae gradatae $8 / 9$ vel $8 / 10$, totae virides. 
In ala posteriore venulae gradatae $6 / 8$ vel $6 / 9$, totae virides.

Sur l'exemplaire type les deux points postérieurs du cinquième segment sont très effacés. D'autres exemplaires pourraient fort bien avoir des points sur chaque segment de l'abdomen.

J'ajouterai que, comme dans les variétés respersa et amabilis de $\boldsymbol{C}$. prasina, ces points ne sont généralement plus appréciables sur des individus desséchés. J'ai étudié cette variété sur l'insecte vivant.

Le prothorax a sur sa région dorsale quatre points : les postérieurs allongés sont aussi marqués que les antérieurs et paraissent tendre à se fusionner avec ceux-ci pour former deux bandes.

Cette variété a la taille du type et des autres formes déjà décrites.

Habitat : Saint-Martin-de-la-Coudre (Charente-Inférieure), 15 aout 1913. Le type dans ma collection.

\section{Description d'une nouvelle Catocala de Syrie [Lep. Noctuidae]}

\section{par J. Vincent.}

Catocala delicata, n. sp. - Envergure 40 mill. ; ailes supérieures allongées, apex à angle aigu.

Ailes supérieures : Fond gris brunâtre clair assez uni, sur lequel ressort très finement écrites en brun foncé les lignes médianes. Ligne basilaire fortement marquéeà la côte formant un angle externe sur la nervure costale et deux autres rentrant sur les bords de la cellule au delà de laquelle elle se termine par un léger trait brun qui n'atteint pas le bord interne. L'anté-médiane, oblique,simple, marquée plus fortement à la côte, formant deux festons ayant les pointes s'infléchissant vers l'extérieur. La post-médiane, dentée de cinq dents

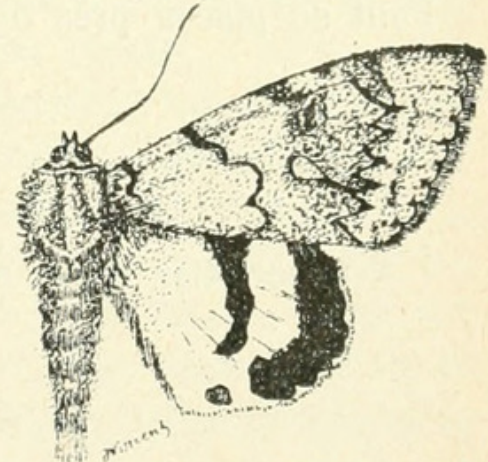

Fig. 1. - Catocala delicata Vincent. aux pointes fines, un peu plus marquée à la còte où elle débute par une tache brune, diffuse, exactement audessus de la réniforme, réunie à la ligne dentée par un trait très fin suivant la nervure 2. A la $4^{\mathrm{e}}$ dent la tache subréniforme est reliée à la post-médiane par un trait horizontal. Ombre médiane réduite, un peu marquée à la côte. Tache réniforme ovale, brune, finement bordée 


\section{$2 \mathrm{BHL}$ Biodiversity Heritage Library}

Lacroix, J L. 1913. "Chrysopides nouveaux [Nevr.]." Bulletin de la Société entomologique de France 1913, 429-431.

https://doi.org/10.5962/bhl.part.16311.

View This Item Online: https://www.biodiversitylibrary.org/item/36377

DOI: https://doi.org/10.5962/bhl.part.16311

Permalink: https://www.biodiversitylibrary.org/partpdf/16311

\section{Holding Institution}

Smithsonian Libraries

\section{Sponsored by}

Smithsonian

\section{Copyright \& Reuse}

Copyright Status: NOT_IN_COPYRIGHT

This document was created from content at the Biodiversity Heritage Library, the world's largest open access digital library for biodiversity literature and archives. Visit BHL at https://www.biodiversitylibrary.org. 